\title{
PBAR DECELERATION IN THE FERMILAB MAIN INJECTOR : TUNE-UP STUDIES WITH PROTON BEAM
}

\author{
C. M. Bhat, G. Wu, K. Meisner, B. Chase, S. Assadi”, B. C. Brown, D. Capista, \\ D. Johnson, I. Kourbanis, A.Hahn, S. Pruss, M-J. Yang and S. Mishra, \\ FNAL*, Batavia, IL 60510, USA
}

\begin{abstract}
In this report we present the results of beam dynamics simulations as well as experiments with protons for deceleration from $150 \mathrm{GeV}$ to $8.9 \mathrm{GeV}$ in the Fermilab Main Injector (MI). The simulations are carried out on two different deceleration schemes: deceleration with a fast recovery MI cycle and with a slow recovery cycle. As a proof of principle we have carried out the first successful deceleration using proton beam in the Main Injector from the Tevatron extraction energy of $150 \mathrm{GeV}$ to the Recycler injection energy of $8.9 \mathrm{GeV}$.
\end{abstract}

\section{INTRODUCTION}

Pbar recycling from the Tevatron collider runs is essential for pbar economy as well as to reach the Run-II luminosity goals[1]. During Run II we plan to provide up to $105 \mathrm{pb}-1 /$ week of ppbar luminosity. We need about $1100 \mathrm{~mA}$ of pbars per ppbar stores in the Tevatron, $60 \%$ of which comes from the pbar source and the remaining $40 \%$ should come from recycling. Usually at the end of each ppbar stores in the Tevatron about $70 \%$ of the pbar beam will survive and are wasted by dumping them to prepare for the next store. We can re-use the pbars by decelerating them from $1 \mathrm{TeV}$ to $150 \mathrm{GeV}$ in the Tevatron and from $150 \mathrm{GeV}$ to $8.9 \mathrm{GeV}$ in the MI, finally storing and cooling in the newly built Recycler Ring (RR)[2] till the beam is needed for the next collider shot. In this report we investigate the beam deceleration only in the MI. MI

There are two issues of concern in beam deceleration in

1) Longitudinal beam dynamics issues: Typically the beam bunches from the Tevatron at $150 \mathrm{GeV}$ are 3-4 eV-s in $53 \mathrm{MHz}$ rf buckets. But the MI acceptance at transition is only $0.5 \mathrm{eV}$-s. Therefore we can not decelerate beam in the MI from $150 \mathrm{GeV}$ to $8.9 \mathrm{GeV}$ without any further rf manipulations.

2) Transverse beam dynamics issues: The dipoles, (and dipole correctors), quadrupoles (quadrupole correctors) and sextupoles have hysteresis. Hence the up-ramp BH-curves are not same as that of down-ramp. Also, the deceleration BHcurves for quadrupole and sextupoles depends upon the final set values of tune and chromaticity.

\footnotetext{
* Work supported by the U.S. Department if Energy under contract No. DE-AC02-76CH03000.
}

In this connection we have studied two schemes- one with a fast recovery $($ a $6 \mathrm{sec}$ ) MI deceleration cycle (schemeA) and with a slow recovery (a $20 \mathrm{sec}$ ) cycle (scheme-B). In scheme-A the pbar in MI will be decelerated from 150 $\mathrm{GeV}$ to $8.9 \mathrm{GeV}$ using $53 \mathrm{MHz}(\mathrm{h}=588)$ rf system. At 8.9 $\mathrm{GeV}$ the beam is transferred to $2.5 \mathrm{MHz}(\mathrm{h}=28) \mathrm{rf}$ buckets and finally transferred to the RR. In scheme-B the beam is initially decelerated to about $25 \mathrm{GeV}$ using $\mathrm{h}=588$ system and the adiabatically transferred to $\mathrm{h}=28$ system and decelerated slowly to $8.9 \mathrm{GeV}$ and finally to the RR. The MI dipole ramps used for these two schemes are shown in Fig.1. Preliminary results of the calculations and experiment carried out on these two schemes are reported previously elsewhere[3].

\section{SIMULATIONS}

Longitudinal beam dynamics simulations have been carried out using ESME[4] for both the cases discussed

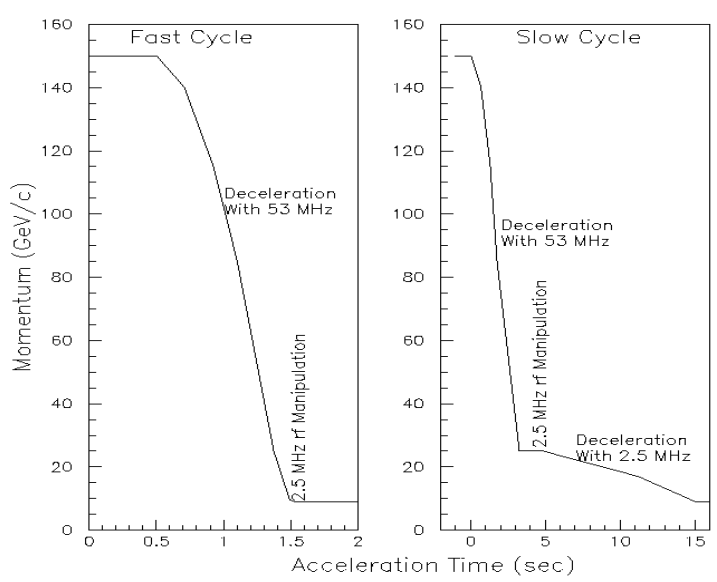

Figure 1. MI Ramps for scheme-A (left) and scheme-B (right) deceleration scenarios.

here by employing the MI parameters from ref.5. We assumed the beam particle distribution is parabolic in $\mathrm{dE}$ $\mathrm{dt}$ space, where $\mathrm{E}$ is the synchronous energy of the beam particles and $t$ is the time. The $53 \mathrm{MHz}$ bunches from the Tevatron come in a train of four bunches with a bunch separation of twenty one $53 \mathrm{MHz}$ bunches. In our calculations we have assumed that a single bunch from the Tevatron injected in to the MI at $150 \mathrm{GeV}$. 
Simulations have been carried out for both the schemes $\mathrm{A}$ and $\mathrm{B}$ [3]. The scheme-A results in a significant longitudinal emittance dilution. Further we have also seen beam loss at transition in the MI with scheme-A in contrast with scheme-B. Here we present the results for better of the two schemes. The figure 2 shows the simulated time evolution of phase-space distribution of particles for the scheme-B. The matching rf voltage between Tevatron and $\mathrm{MI}$ for $4 \mathrm{eV}$-s beam is about 0.4 MV in the MI $53 \mathrm{MHz}$ rf system, which is only $10 \%$ of the maximum rf voltage attainable. As a result of this we can afford to decelerate the $53 \mathrm{MHz}$ bunches arriving from the Tevatron to $25 \mathrm{GeV}$ (very close to transition energy of $20.49 \mathrm{GeV}$ ) in the MI with out any problem. At $25 \mathrm{GeV}$ the beam bunches are transferred to $\mathrm{h}=28 \mathrm{rf}$ system and beam is decelerated to $8.9 \mathrm{GeV}$. Unfortunately, we do not have more than $80 \mathrm{kV}$ available from our $\mathrm{h}=28 \mathrm{rf}$ system. As a results of this we need to decelerate very slowly. The minimum $\mathrm{dp} / \mathrm{dt}$ during the deceleration between 25 $\mathrm{GeV}$ to $8.9 \mathrm{GeV}$ is selected to be $-1.7 \mathrm{GeV} / \mathrm{c} / \mathrm{sec}$. In the simulation we see very small emittance growth through the entire deceleration process.
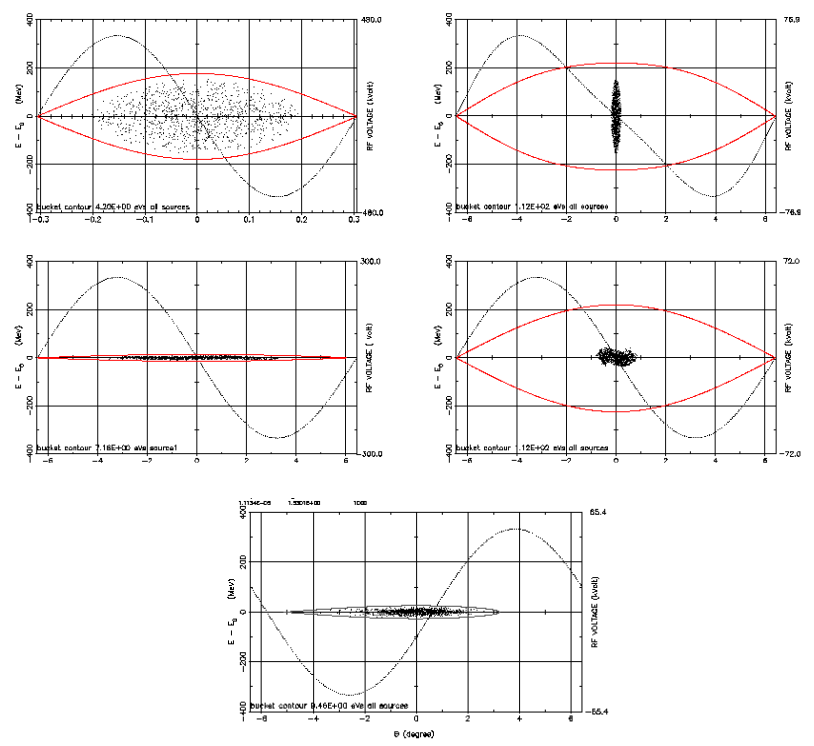

Figure.2: ESME simulations for the slow deceleration ramp. $\mathrm{dE}$ vs $\mathrm{d} \phi$ for the particle distributions are shown in each display. The closed contours represent buckets and the sinusoidal curve represent the rf voltage wave form. Top left: beam bunch in $\mathrm{h}=588$ bucket at $150 \mathrm{GeV}$, top right: bunch in $\mathrm{h}=28$ bucket at $25 \mathrm{GeV}$, middle-row left: bunch in $\mathrm{h}=28$ bucket at $25 \mathrm{GeV}$ after bunch rotation, middle-row right: at $25 \mathrm{GeV}$ after bunch squeezing, bottom: beam in $\mathrm{h}=28$ bucket at $8.9 \mathrm{GeV}$ before injection into the Recycler Ring.

Thus the beam dynamics calculations showed that slow deceleration is more favorable than the fast deceleration from the point of view of transfer efficiency as well as emittance preservation. However, the slow deceleration in the MI needs hardware as well as software developments which are in progress.

\section{PROTON DECELERATION IN THE MI FROM 150 GEV TO 8.9 GEV}

The beam deceleration experiments are conducted in the MI using protons from the Fermilab Booster and accelerating it to $150 \mathrm{GeV}$ and the ramp developed for the scheme-B. In the absence of $2.5 \mathrm{MHz}$ phase control system, we ended up in conducting all of our deceleration experiments using only the $\mathrm{h}=588$ system.

Table 1: Measured longitudinal emittance on the deceleration ramp.

\begin{tabular}{|l|l|l|}
\hline & Energy $(\mathbf{G e V})$ & Emittance \\
\hline Flat top & 150 & $0.4 \mathrm{eVs}$ \\
\hline Back-porch & 25 & $0.4 \mathrm{eVs}$ \\
\hline $\begin{array}{l}\text { RR- Injection } \\
\text { enrgy }\end{array}$ & 8.9 & $0.8 \mathrm{eVs}$ \\
\hline
\end{tabular}

A controlled beam deceleration in MI is in general not trivial because of the issues discussed earlier. Further, LLRF control needs to be modified to allow phase jump on -ve side of the rf wave at transition. Accelerator control program to correct the orbits, tunes and chromaticities[6] were also modified to accommodate deceleration ramp.

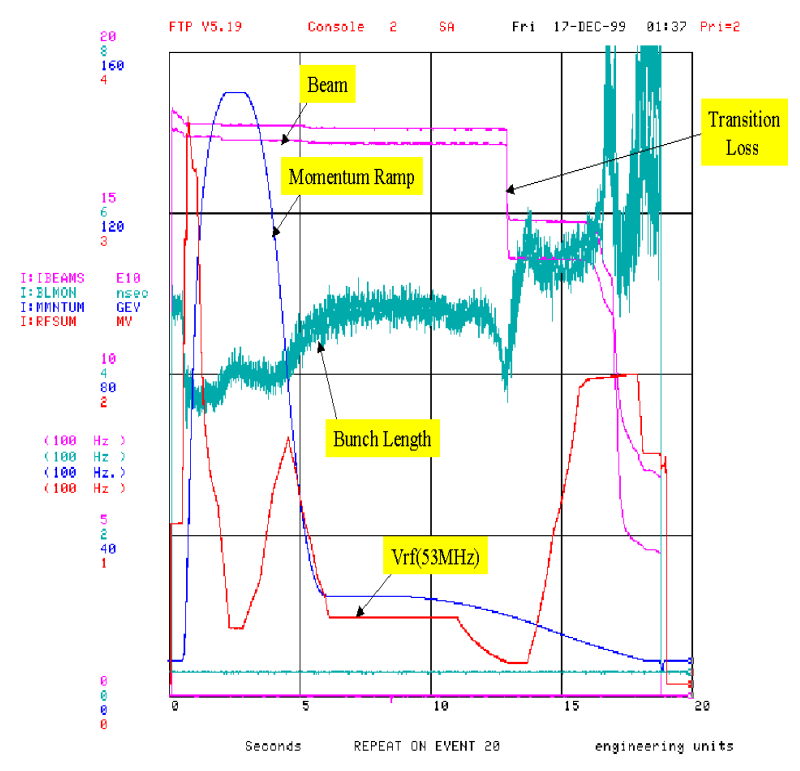

Figure 3: BEAM- MI beam intensity in units of E10, momentum $(\mathrm{GeV})$, bunch length (nsec) and $\operatorname{Vrf}(53 \mathrm{MHz})-$ rf voltage on $53 \mathrm{MHz}$ rf system (MV) as a function of time $(\mathrm{sec})$. 
The Fig. 3 represents typical data taken during proton beam deceleration in the MI with scheme-B (slightly modified, and deceleration carried out with $53 \mathrm{MHz}$ system) after correcting the orbit and the magnet hysteresis up to about $15 \mathrm{GeV}$. The early part of the data represents the beam acceleration from $8.9 \mathrm{GeV}$ to 150 $\mathrm{GeV}$. The data shown contains Beam (scale: 0 - 20E10), Momentum P(scale:0 to $160 \mathrm{GeV} / \mathrm{c}$ ) Bunch length (scale: 0-8 nsec) and voltage on $53 \mathrm{MHz}$ rf system during the operation (scale: 0 to $4 \mathrm{MV}$ ). Almost $100 \%$ of the beam survive from $150 \mathrm{GeV}$ (flat-top) to $20.49 \mathrm{GeV}$ (down) (transition energy). Below the transition energy $85 \%$ of the beam survive. We saw another $50 \%$ beam loss at about $13.5 \mathrm{GeV}$ leaving finally about $40 \%$ of the beam to $8.9 \mathrm{GeV}$. Table 1 shows the measured longitudinal emittance of the beam for the entire process. We have used bunch length monitor data and correct phase angle at $8.9 \mathrm{GeV}, 150 \mathrm{GeV}$, and at $25 \mathrm{GeV}$ to estimate the longitudinal emittance.

The data clearly shows that very small emittance growth from $150 \mathrm{GeV}$ to transition energy. At transition, however, we observe beam loss. This beam loss can be understood as follows: The non-adiabatic time, Tc, for the case studied here is about $20 \mathrm{msec}$. The maximum $(\mathrm{dp} / \mathrm{p})_{99 \%}$ corresponding to the MI admittance of $0.5 \mathrm{eVs}$ [1] is $0.9 \%$. But, the (dp/p) ${ }_{99 \%}$ (beam) corresponding to the bunch length of $4 \mathrm{nsec}$ ( from the figure 3 ) near transition energy is about $1.1 \%$. Hence, the beam loss seen near transition is natural. About $15 \%$ beam loss is observed under present conditions. This problem can be eliminated if we use $2.5 \mathrm{MHz}$ rf buckets instead of $53 \mathrm{MHz}$ rf for deceleration through the transition. Second beam loss occurring at $13.5 \mathrm{GeV}$ is not fully understood at this time. Our calculations shows that it is not due to limited rf bucket area. The actual cause may be arising from the magnet power supplies switch off as we decelerate the beam which was not emphasized so far during the beam operation.
A simple extrapolation of the results of the experiment to a case of deceleration with $\mathrm{h}=28$ system (as proposed in ref. 2) with about $60 \mathrm{kV}$ of $\mathrm{rf}$ voltage suggests that one should be able to decelerate a bunch with longitudinal emittance as big as $7 \mathrm{eV}$-sec through MI transition without any beam loss.

We have also measured transverse emittance using flying wires of MI. Data showed no transverse emittance growth during the deceleration.

In summary, we have conducted series of pbar deceleration simulations as well as experiments using proton beam in the MI. This effort was the first attempt of proof of principle for decelerating the beam in the MI. We were able to successfully decelerate the beam from 150 $\mathrm{GeV}$ to $8.9 \mathrm{GeV}$ with about $100 \%$ till transition energy and $85 \%$ beyond. This result is consistent with our longitudinal beam dynamics simulations. But, needs further improvements, which are being working on.

One of the authors (CMB) would like to thank Jim MacLachlan for many useful discussions during simulation using ESME. We would like to thank the MCR operation group for their cooperation during the beam study.

\section{REFERENFES}

\# Presently at ORNL

[1] "Fermilab Run II Hand Book, 1995 (unpublished).

[2] The Fermilab Recycler Ring Technical Design Report, G. Jackson, Fermilab TM-1991

[3] C. M. Bhat, MI note 271(2000); C.M.Bhat and J. MacLachlan, IEEE, PAC97, p 1590

[4] J. MacLachlan, ESME2000, FERMILAB TM-2132.

[5] Fermilab Main Injector Technical Design Hand Book, 1994, and Run II Hand Book

[6] C.M. Bhat et al., PAC1997, Vancouver, p 3242 